\title{
ACCIÓN ESTATAL PARA LA PREVENCIÓN DEL TRABAJO INFANTIL EN CORRIENTES: PROGRAMA CRECER SIN TRABAJO INFANTIL
}

\author{
Juan Carlos Díaz* - María Alejandra Henderson**
}

\section{Introducción}

El Trabajo Infantil es conceptualizado por el Plan Nacional para la Erradicación del Trabajo Infantil de la CONAETI como:

Toda actividad económica y/o estrategia de supervivencia, remunerada o no, realizada por niñas y niños, por debajo de la edad mínima de admisión al empleo o trabajo, o que no han finalizado la escolaridad obligatoria o que no han cumplido los 18 años si se trata de trabajo peligroso.

En relación con las políticas públicas orientadas a alcanzar la erradicación del trabajo infantil y la efectiva protección de los adolescentes que trabajan, y ante la falta de unanimidad en cuanto al tratamiento jurídico de la problemática del Trabajo Infantil es por lo que tanto concepciones legislativas como doctrinarias oscilan entre asignar al Trabajo Infantil dos Corrientes de Pensamiento para su tratamiento: 1) Abolicionista: proponiendo la erradicación total del Trabajo Infantil: con basamento en que todo trabajo de menores de 18 años está prohibido, y 2) Reglamentarista: corriente que responde a nuestro sistema legislativo en materia de derecho laboral, haciendo una diferencia en grupos etarios para los cuales dependiendo de la edad, para unos va a estar absolutamente prohibido, proponiendo su erradicación y para otros propone su efectiva protección ante posibles abusos.

Habiendo realizado una investigación sobre el tema en cuestión, y luego de una reflexión llevada a cabo en un tiempo de desarrollo del análisis de la información obtenida, este equipo de investigación optó por tomar la posición reglamentarista que coincide con la tomada por el Estado Argentino.

\footnotetext{
*Abogado. Prof. libre de Derecho del Trabajo, Cátedra a cargo del Dr. César A Vallejos, de la Facultad de Derecho y Ciencias Políticas de la UNNE.

**Abogada. Prof. libre de Derecho del Trabajo, Cátedra a cargo del Dr. César A Vallejos, de la Facultad de Derecho y Ciencias Políticas de la UNNE.
} 


\section{Antecedentes}

A comienzos del siglo XVI, los niños y las niñas ocupaban un rol social similar a los adultos, trabajaban como ellos y no tenían un estatus particular. A partir de los siete años de edad se los incorporaba al mundo del trabajo en su comunidad, donde el trabajo y la familia eran considerados como una unidad productiva.

En el siglo XVII, con el advenimiento del capitalismo, la situación de la niñez empezó a ser pensada en forma diferenciada del adulto, dándole mayor valor a su rol social. Se le dio a la educación un papel fundamental y tanto las familias como la escuela comenzaron a ser consideradas importantes para el desarrollo de los niños. A pesar de esta visión, seguía habiendo grandes diferencias en la infancia según las condiciones económicas, culturales y sociales; los niños de los sectores populares seguían trabajando en minas, industria textil y agricultura, mientras que los de familias burguesas gozaban de cuidado y protección.

A partir de la Revolución Industrial y durante el siglo XIX, los niños de las familias pobres representan un claro eslabón en el proceso productivo, determinándose que las actividades fueran realizadas de un modo acorde con su fuerza y capacidad.

Recién en el siglo XX, con el advenimiento del "derecho protector", se reconoce al trabajo infantil como una problemática a tener en cuenta por los riesgos que conlleva.

Las primeras leyes reglamentan el trabajo de mujeres y menores (Leyes $\mathrm{N}^{\circ}$ 5291 y $^{\circ} 11317$ ) y se hacen extensivas al ámbito rural, sin prohibir el trabajo infantil en el marco de una explotación familiar.

La consideración especial hacia la infancia surge con la Declaración de los Derechos del Niño, aprobada por la Asamblea General de las Naciones Unidas en 1959, promulgándose la vulnerabilidad especial del niño/a y remarcando la necesidad de darles una protección especial para su desarrollo y una vida en condiciones de libertad y dignidad. Se toma conciencia de que el trabajo infantil rompe con el concepto de infancia como período que permite un desarrollo personal, una formación educativa y una integración positiva a la sociedad

En 1989 nuestro país adopta la Convención sobre los Derechos del Niño que convierte a los niños en sujetos de derechos, comprometiéndose a que se respetará y asegurará su aplicación sin distinción alguna. Dicha proclama, más la promulgación de la ley sobre la Prohibición del Trabajo Infantil y la Protección del Trabajo Adolescente (Ley $N^{\circ}$ 26390), constituyen tal vez las herramientas 
más importantes en pos de su erradicación.

A pesar del progreso de las leyes laborales y la adhesión a la proclama de derechos del niño, el trabajo infantil aún hoy es preocupante y su erradicación requiere el control permanente de políticas públicas y el compromiso de toda la comunidad.

\section{El trabajo infantil}

El término trabajo infantil se refiere a cualquier trabajo que es física, mental, social o moralmente perjudicial o dañino para el niño y afecta su escolaridad, al privarlo de la oportunidad de ir a la escuela, obligarlo a abandonar prematuramente las aulas o exigirle que combine la asistencia a la escuela con largas jornadas de labor.

El trabajo infantil es aquel que se realiza por debajo de la edad mínima de admisión al empleo fijada en cada país y que no puede ser inferior a la edad en que cesa la educación obligatoria. En Argentina cualquier trabajo realizado por personas menores de 16 años está prohibido.

También se consideran trabajo infantil aquellas actividades económicas realizadas por debajo de los 18 años que con independencia de que interfieran en la escolarización, se realizan en ambientes peligrosos o en condiciones que afecten el desarrollo de los niños, niñas y adolescentes. Las formas más extremas de trabajo infantil son aquellas que esclavizan al niño, lo separan de su familia, lo exponen a graves peligros y enfermedades, o lo dejan abandonado a su suerte en la vía pública, en muchos casos, desde temprana edad.

Existen diferencias considerables entre las numerosas formas de trabajo realizadas por niños. Algunas son dificiles y exigentes, otras, más peligrosas e incluso reprobables desde el punto de vista ético. En el marco de su trabajo, los niños realizan una gama muy amplia de tareas y actividades.

No todas las tareas realizadas por los niños deben clasificarse como trabajo infantil que se ha de eliminar. Por lo general, la participación de los niños o los adolescentes en trabajos que no atentan contra su salud y su desarrollo personal ni interfieren con su escolarización se considera positiva.

Entre otras actividades, cabe citar la ayuda que prestan a sus padres en el hogar, la colaboración en un negocio familiar o las tareas que realizan fuera del horario escolar o durante las vacaciones para ganar dinero de bolsillo. Este tipo 
de actividades son provechosas para el desarrollo de los pequeños y el bienestar de la familia; les proporcionan calificaciones y experiencia, y les ayuda a prepararse para ser miembros productivos de la sociedad en la edad adulta.

El término "trabajo infantil" suele definirse como todo trabajo que priva a los niños de su niñez, su potencial y su dignidad, y que es perjudicial para su desarrollo físico y psicológico.

Se alude al trabajo que:

- es peligroso y prejudicial para el bienestar físico, mental o moral del niño;

- interfiere con su escolarización puesto que:

- les priva de la posibilidad de asistir a clases;

- les obliga a abandonar la escuela de forma prematura, o

- les exige combinar el estudio con un trabajo pesado y que insume mucho tiempo.

En las formas más extremas de trabajo infantil, los niños son sometidos a situaciones de esclavitud, separados de su familia, expuestos a graves peligros y enfermedades y/o abandonados a su suerte en la calle de grandes ciudades (con frecuencia a una edad muy temprana). Cuándo calificar o no de "trabajo infantil" a una actividad específica dependerá de la edad del niño o la niña, el tipo de trabajo en cuestión y la cantidad de horas que le dedica, las condiciones en que lo realiza, y los objetivos que persigue cada país. La respuesta varía de un país a otro y entre uno y otro sector.

La CONAETI entiende que trabajo infantil es:

Toda actividad económica y/o estrategia de supervivencia, remunerada o no, realizada por niñas y niños, por debajo de la edad mínima de admisión al empleo o trabajo (16 años), o que no han finalizado la escolaridad obligatoria o que no han cumplido los 18 años si se trata de trabajo peligroso.

\section{Las peores formas de trabajo infantil}

Aunque el trabajo infantil adopta muchas formas diferentes, una prioridad es la eliminación inmediata de sus peores formas. Según la definición del artículo 3 del Convenio núm. 182 de la OIT: a) todas las formas de esclavitud o las prácticas análogas a la esclavitud, como la venta y la trata de niños, la servidumbre por 
deudas y la condición de siervo, y el trabajo forzoso u obligatorio, incluido el reclutamiento forzoso $\mathrm{u}$ obligatorio de niños para utilizarlos en conflictos armados; b) la utilización, el reclutamiento o la oferta de niños para la prostitución, la producción de pornografía o actuaciones pornográficas; c) la utilización, el reclutamiento o la oferta de niños para la realización de actividades ilícitas, en particular la producción y el tráfico de estupefacientes, tal como se definen en los tratados internacionales pertinentes, y; d) el trabajo que, por su naturaleza o por las condiciones en que se lleva a cabo, es probable que dañe la salud, la seguridad o la moralidad de los niños.

El trabajo que pone en peligro el desarrollo, físico, mental o moral del niño, sea par su naturaleza o por las condiciones en las que se efectúa, es denominado "trabajo peligroso".

\section{El Trabajo Infantil en Argentina}

Según la OIT, cerca de 215 millones de NNyA trabajan en el mundo. Más de la mitad están expuestos a las peores formas de trabajo infantil como trabajo en ambientes peligrosos, esclavitud, y otras formas de trabajo forzoso, actividades ilícitas incluyendo el tráfico de drogas y prostitución, así como su participación involuntaria en los conflictos armados.

En América Latina se estima que el trabajo infantil afecta a aproximadamente 14 millones de NNyA entre los 5 y los 17 años. Mientras que en la Argentina, se estimaba en 2004 que $6,5 \%$ de los niños y niñas entre 5 y 13 años trabajan al menos una hora por semana y $20,1 \%$ de los/as adolescentes entre 14 y 17 años (aproximadamente 456.207 NNyA).

El trabajo infantil se presenta como una problemática pues constituye una vulneración de los derechos fundamentales de la niñez, trayendo como consecuencia perjuicios para el desarrollo físico, psíquico y social del niño o la niña. Se constituye en una cuestión social, es decir es un hecho social que no sólo afecta a los propios niños y niñas que trabajan, sino que confronta con un proyecto de país y compromete a todos sus ciudadanos.

Además es una problemática compleja en el sentido que confluyen y se interrelacionan condicionantes económicos, políticos, legales y culturales. Por lo tanto, el trabajo infantil como problemática social 
compleja demanda la puesta en marcha de políticas y acciones concretas, pero también requiere una sensibilización y concientización de la sociedad.

\section{Normativa vigente en la República Argentina al respecto}

En la Argentina está prohibido el trabajo de los niños por debajo de los 16 años, de acuerdo a la Ley $\mathrm{N}^{\circ} 26390$ de Prohibición del Trabajo Infantil y Protección del Trabajo Adolescente [http://www.trabajo.gov.ar/conaeti/normativa.asp].

Considerar las estrategias de supervivencia como trabajo implica ampliar el concepto de trabajo establecido por la legislación laboral incluyendo modalidades de índole informal tales como las desarrolladas en la vía pública: (cirujeo, cartoneo, mendicidad). En la agricultura: ya sea en unidades productivas familiares o para terceros. En el ámbito doméstico: tanto el realizado para terceros como el realizado dentro del hogar.

Las modalidades consideradas como peores formas de trabajo infantil son: explotación sexual comercial infantil, utilización de niñas y niños para el tráfico de estupefacientes, su utilización en pornografia, toda forma de esclavitud y todo trabajo que por su naturaleza o circunstancias en las que se realiza atente contra la salud, la seguridad o la moral de niños y niñas. Estas formas, que implican delito, son entendidas también como trabajo infantil considerándose a la niña o el niño que las realiza "víctimas", siendo el explotador sobre quién recae la sanción penal.

Las modalidades de trabajo infantil peligroso, penoso, e insalubre son también consideradas peores formas de trabajo infantil. La Ley de Contrato de Trabajo explicita que está prohibido ocupar a menores de dieciocho (18) años de edad en los trabajos que revistan estas características (artículos 176 al 191). Son tareas penosas las actividades que demandan esfuerzos físicos y psíquicos extraordinarios y las que resultan aflictivas por el entorno en que se realizan o por la propia clase de prestación. Son tareas peligrosas las que comprometen la seguridad e integridad psico-física de quien las ejecuta. Finalmente, las tareas insalubres son las que provocan daños en la salud de los seres humanos.

\section{Políticas públicas que se han implementado en Argentina en relación con la erradicación del trabajo infantil}

Las políticas públicas que se han implementado en relación con la erradi- 
cación del trabajo infantil, abarcan en el país la sanción de normas y la implementación de programas y acciones, incluyendo la creación de instituciones específicas, no sólo a nivel nacional sino también en las distintas provincias del país.

A nivel internacional, el marco normativo vigente sobre trabajo infantil cuenta con tres instrumentos: el Convenio sobre la edad mínima de admisión al empleo, 1973 (núm. 138) y el Convenio sobre las peores formas de trabajo infantil, 1999 (núm. 182) ambos de la OIT y ratificados por Argentina, y la Convención sobre los Derechos del Niño (CDN), que además de su ratificación fue incorporada a la Constitución Nacional en 1994. Esto habilitó una sucesión de avances legislativos, que tiene como acción más reciente, en 2013, el establecimiento de sanciones penales a quienes obtengan un beneficio económico del trabajo de niños, niñas y adolescentes.

En cuanto a políticas y acciones que promueven el cumplimiento efectivo de las normas, han sido desarrolladas a través de instituciones públicas y privadas.

Dentro del ámbito público la Comisión Nacional para la Erradicación del Trabajo Infantil (CONAETI), compuesta por un conjunto de ministerios nacionales, organizaciones de empleadores, de trabajadores y el Episcopado estableció el marco de una política con alcance nacional a través del Plan Nacional para la Prevención y Erradicación del Trabajo Infantil. La CONAETI, a su vez, impulsó junto con el Consejo Federal del Trabajo (CFT), la creación de las Comisiones Provinciales de Erradicación del Trabajo Infantil (COPRETIS), que trabajan articulando recursos locales, provinciales y nacionales. La cercanía de estas comisiones provinciales con los problemas locales permite adecuar las políticas a las distintas realidades territoriales.

\section{El trabajo adolescente}

El trabajo adolescente se refiere a la franja etaria de 16 a 18 años. La normativa nacional protege el trabajo adolescente, que se encuentra regulado en el Capítulo VIII de la Ley de Contrato de Trabajo, y rige la prohibición absoluta de realizarse en ambientes o condiciones peligrosas, penosas o insalubres. Además, la jornada no debe exceder las 6 horas diarias y se prohíbe la realización de horas extras y el horario nocturno.

Los derechos de nuestros niños, niñas y adolescentes se encuentran contenidos en la Constitución Nacional, la Convención sobre los Derechos del Niño, 
los Convenios de la Organización Internacional del Trabajo (OIT) $\mathrm{N}^{\circ} 138 \mathrm{y}$ $N^{\circ} 182$ sobre "La Edad Mínima de Admisión al Empleo", ratificado por Ley $\mathrm{N}^{\circ} 24650$, y sobre "las peores formas de trabajo infantil", ratificado por la Ley $\mathrm{N}^{\circ} 25255$, por la Ley $\mathrm{N}^{\circ} 26061$ de Protección Integral de los Derechos de las Niñas, Niños y Adolescentes, entre otras normas.

En materia laboral rige, desde junio del 2008, la Ley $N^{\circ} 26390$ sobre Prohibición del Trabajo Infantil y Protección del Trabajo Adolescente, además de otras normas específicas según la actividad, como ser la Ley № 26727 de 2011 sobre Régimen de Trabajo Agrario.

\section{Principales causas del trabajo infantil en Argentina}

Los datos existentes en Argentina permiten advertir el estrecho vínculo entre pobreza y trabajo infantil. La insuficiencia de servicios Trabajo Infantil y Protección Social en Argentina Preguntas y Respuestas básicos también puede traducirse en que los niños deban realizar un mayor número de tareas, como la recolección de leña o agua.

Son relevantes también, el nivel educativo de las madres y el acceso al sistema educativo de los niños y adolescentes que trabajan. Pero la vulnerabilidad económica no es la única causa del trabajo infantil, las creencias y valoraciones que permiten su naturalización, ya sea porque se lo percibe como inevitable, como estrategia de supervivencia de los sectores pobres de la sociedad, o porque se lo considera como algo positivo asociado al valor formativo del trabajo en la niñez, indican la existencia de condicionantes culturales que justifican el trabajo infantil y que impiden visualizarlo como un problema que debe ser combatido.

Existen diferentes condicionantes del trabajo infantil tales como el económico, que impulsa a muchas familias a utilizar toda la fuerza laboral para lograr la subsistencia. Pero también existen condicionantes culturales, por ejemplo para el trabajo infantil artístico que vincula el trabajo con modelos sociales de éxito, o en el ámbito rural que se lo considera una "tradición cultural" muy arraigada y dificil de modificar. Los trabajos que realizan niños y niñas afectan su salud psíquico-física y les impiden el desarrollo de su infancia, limitan su tiempo de juego y de descanso e interfieren en su educación. Ninguna forma o modalidad de trabajo infantil es formativa. 
Lo que se debe hacer ante situaciones detectadas de trabajo infantil

El Estado argentino aborda esta problemática desde el marco establecido por el Plan Nacional para la Prevención y Erradicación del Trabajo Infantil. El objetivo de este instrumento diseñado e implementado por la CONAETI de manera consensuada con los organismos provinciales pertinentes, es prevenir y erradicar el trabajo infantil en todas sus formas, mediante acciones que favorezcan la participación de los distintos actores sociales en todo el país.

El Plan establece una modalidad de intervención multisectorial, articulando acciones con los sectores empresario y sindical, y con organizaciones de la sociedad civil y organismos de gobierno.

Ante situaciones de trabajo infantil puede contactarse en el ámbito nacional con la CONAETI: Av. Leandro N. Alem 628. CABA. Correo electrónico: [conaeti@trabajo.gob.ar].

En el ámbito provincial hay que dirigirse a la COPRETI correspondiente. En Corrientes la Comisión Provincial de Prevención y Erradicación Progresiva del Trabajo Infantil se encuentra en Calle San Juan 1317, Ciudad de Corrientes.

\section{Programa crecer sin trabajo infantil}

\section{Normativa}

La legislación de nuestro país juega un papel imprescindible para erradicar el trabajo infantil. La armonización legislativa consiste en adecuar nuestra normativa a los convenios internacionales aprobados y ratificados por Argentina.

La problemática del trabajo infantil está íntimamente vinculada con la Convención de los Derechos del Niño, que adquirió jerarquía constitucional, y con los Convenios $\mathrm{N}^{\mathrm{o}} 138$ y N $\mathrm{N}^{\mathrm{o}} 182$ de la OIT, aprobados por las Leyes $\mathrm{N}^{\mathrm{o}}$ $24650 \mathrm{y} \mathrm{N}^{\circ} 25255$ respectivamente.

La normativa que regula (prohíbe) el trabajo infantil en la República Argentina es un tema que debe analizarse en conjunto, pero no debe olvidarse que en el derecho del trabajo cobran particular relieve determinadas fuentes propias, como la autonomía negocial colectiva (convenios colectivos) y el reglamento interno de los establecimientos.

Existen otras numerosas leyes o estatutos especiales que rigen para determinada categoría de trabajadores, regulando distintas profesiones. Estas normas 
particulares se dictaron exclusivamente para determinados sectores laborales y constituyen la fuente formal del contrato y la relación de trabajo.

\section{Comisión Nacional para la Erradicación del Trabajo Infantil (CONAETI)}

La Comisión Nacional para la Erradicación del Trabajo Infantil (CONAETI) tiene por función coordinar, evaluar y dar seguimiento a los esfuerzos en favor de la prevención y erradicación real y efectiva del trabajo infantil.

El 5 de diciembre de 1996 el Ministerio de Trabajo y Seguridad Social de la República Argentina (actualmente Ministerio de Trabajo, Empleo y Seguridad Social), suscribió un "Memorando de Entendimiento" con la Organización Internacional del Trabajo (OIT), organismo que propone cómo metodología de abordaje frente al trabajo infantil la conformación de mesas intersectoriales con organismos gubernamentales y no gubernamentales para aunar esfuerzos en torno a esta compleja problemática social, que no puede ser abordada eficazmente por un solo sector.

La CONAETI se creó siguiendo estas pautas en el ámbito del Ministerio de Trabajo el 25 de agosto de 2000, mediante el Decreto $\mathrm{N}^{\circ}$ 719/00. Es una comisión de carácter interministerial e intersectorial presidida por el Ministerio de Trabajo, Empleo y Seguridad Social. Sus acciones están dirigidas fundamentalmente a propiciar desde todos los ámbitos y niveles de intervención la prevención y erradicación del trabajo infantil.

\section{Comisiones Provinciales para la Prevención y Erradicación del Trabajo Infantil (COPRETI)}

En el marco del Convenio celebrado entre el Ministerio de Trabajo, Empleo y Seguridad Social, la Comisión Nacional para la Erradicación del Trabajo Infantil (CONAETI) y el Consejo Federal del Trabajo, se crearon las comisiones provinciales para la erradicación del trabajo infantil (COPRETI) para coordinar las actividades que se implementen en las jurisdicciones. Actualmente se trabaja en forma coordinada con 23 comisiones provinciales. 


\section{Crecer sin Trabajo Infantil-Provincia de Corrientes}

El programa "Crecer sin Trabajo Infantil" de la Dirección de Empleo de la Subsecretaría de Trabajo, dependiente del Ministerio de Industria, Trabajo y Comercio; tiene como objetivo general disminuir progresivamente el Trabajo Infantil realizado por Niñas y Niños y proteger el Trabajo Adolescente en la Provincia de Corrientes, mediante acciones de prevención, concientización y detección.

Corrientes, Ciudad de Corrientes. Comisión Provincial de Prevención y Erradicación Progresiva del Trabajo Infantil. Domicilio: San Juan 1317. Email: [crecersintrabajoinfantil@live.com], [subsectrabajoctes@hotmail.com].

\section{Consejo Provincial de Prevención y Erradicación Progresiva del Trabajo Infantil}

En este marco la sala de Sesiones de la Honorable Legislatura de la Provincia de Corrientes, el día 12 de julio de 2006 sanciona la Ley $\mathrm{N}^{\circ} 5724$ que crea el Consejo Provincial de Prevención y Erradicación Progresiva del Trabajo Infantil (COPRETI).

\section{Ley $\mathbf{N}^{\circ} 5724$}

Consejo Provincial de Prevención y Erradicación Progresiva del Trabajo Infantil (COPRETI)

Artículo $1^{\circ}$ : Créase, en el ámbito de la Subsecretaría de Trabajo, el Consejo Provincial de Prevención y Erradicación Progresiva del Trabajo Infantil (COPRETI), EL Consejo tendrá funciones de asesoramiento, seguimiento de la ejecución y monitoreo de la gestión.

El Consejo creado por esta Ley remplazará a la Comisión creada por Decreto 2232/02 y asumirá las funciones establecidas en el Decreto Poder Ejecutivo

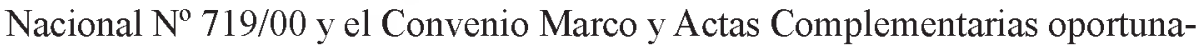
mente firmadas por la Provincia.

Artículo $2^{\circ}$ : El Consejo Provincial de Prevención y Erradicación Progresiva del Trabajo Infantil estará presidido por el Subsecretario de Trabajo e integrado por representantes de los Ministerios de Gobierno, de Salud Pública y de 
Educación y Cultura, de la Secretaria de Desarrollo Humano, del Instituto de la Vivienda de Corrientes, de la Dirección de Minoridad y Familia, de Cáritas Arquidiocesana Corrientes, de APICC, de la Gerencia de Empleo del Ministerio de Trabajo, Empleo y Seguridad Social de la Nación, Delegación Corrientes y de otras organizaciones de la sociedad civil que determine el Decreto reglamentario.

Artículo 3: Créase, en el ámbito de la Subsecretaria de Trabajo, la Dirección Ejecutiva Provincial de Prevención y Erradicación Progresiva del Trabajo Infantil (DEPETI), serán sus funciones la ejecución de programas y líneas de acción, coordinación entre distintas áreas de gobierno y organizaciones de la sociedad civil, evaluación de la población ejecutiva y toda otra inherente a su competencia. Tendrá como consejo asesor al COPRETI creado en el artículo $1^{\circ}$ de la presente Ley y tendrá especial cuidado en tender a mantener al menor dentro del sistema escolar.

Artículo $4^{\circ}$ : La Dirección Ejecutiva estará a cargo del Subsecretario de Trabajo y contará con un área administrativa, un área de inspección y un área de asistentes sociales.

Artículo 5 ${ }^{\circ}$ : Autorícese al Ministerio de Hacienda y Finanzas a realizar las asignaciones y modificaciones presupuestarias que correspondan a los efectos de contratar personal idóneo para cumplir funciones en las áreas detalladas en el artículo anterior.

Artículo $6^{\circ}$ : El COPRETI y la DEPETI podrán celebrar convenios con entidades públicas o privadas para el mejor cumplimiento de los objetivos de la presente ley.

Artículo $7^{\circ}$ : Créase el Fondo Especial para la erradicación del trabajo infantil el que se constituirá con lo recaudado en concepto de multas por violación a la legislación en materia del trabajo de menores, por donaciones de privados, por subsidios otorgados por Entes Descentralizados, todo otro fondo que le fuera otorgado por el Poder Ejecutivo, a través de cualquiera de sus áreas, así como los fondos que remita el Poder Ejecutivo Nacional, a través de cualquiera de sus áreas, con destino a la lucha contra el flagelo del trabajo infantil.

Artículo $8^{\circ}$ : A los efectos de la administración del Fondo Especial creado en el artículo anterior la DEPETI dispondrá de una cuenta en el Banco de Corrientes, de carácter intangible

Artículo $9^{\circ}$ : Los Ministerios y Organismos Descentralizados colaborarán con el COPRETI y con la DEPETI y en especial dispondrán, a su solicitud y en la medida de sus posibilidades, de coberturas de salud, subsidios, planes 
sociales y becas, que sean necesarias para mantener fuera del mercado laboral al menor rescatado de él.

Artículo $1^{\circ}$ : Comuníquese al Poder Ejecutivo.

Fecha de Sanción: 12/07/2006

\section{Conclusión}

La prevención y erradicación del trabajo infantil es una de las metas prioritarias del Estado Argentino. El trabajo infantil constituye una vulneración de los derechos fundamentales de la niñez, trayendo como consecuencia perjuicios para el desarrollo físico, psíquico y social del niño o niña.

No es un hecho aislado, sino que se presenta como una problemática social compleja que requiere la implementación de acciones concretas en distintas materias, entre las que se encuentra el plano normativo.

Cuando la República Argentina ratificó, mediante la Ley $\mathrm{N}^{\circ} 24650$, el Convenio $N^{\circ}$ 138/73 de la OIT, sobre Edad Mínima de Admisión al Empleo se comprometió a seguir una política nacional que asegure la abolición efectiva del trabajo de los niños y niñas y eleve progresivamente la edad mínima de admisión al empleo o al trabajo a un nivel que haga posible su más completo desarrollo físico y mental.

En ese momento histórico, la Argentina, estableció como edad mínima para poder trabajar, los catorce años, haciendo uso de la opción que da el mismo convenio, al entender que su economía y medios de educación estaban insuficientemente desarrollados.

Con el correr del tiempo, el país ha ido superando estas circunstancias, por lo que elevar la edad mínima de acceso al empleo comenzó a emerger como una asignatura pendiente.

Muchos fueron los proyectos legislativos que sostuvieron la necesidad de este cambio, pero fue recién en el año 2008 que podemos celebrar la concreción de este anhelo de quienes bregaron por una niñez libre de trabajo infantil y con trabajo adolescente protegido.

La Ley No 26390 sobre Prohibición del Trabajo Infantil y Protección del Trabajo Adolescente es un salto cualitativo importante en materia de protección de los derechos de nuestros adolescentes trabajadores y de los derechos de nuestros niños y niñas, al ratificar la posición asumida por la República Argentina acerca de la prohibición del trabajo infantil. 


\section{Bibliografía:}

Bordagorry, V. 2011. "Trabajo de mujeres y menores". En Buompadre, J. Revista de la Facultad de Derecho y Ciencias Sociales y Politicas. Nueva Serie. Año 5. No 9. Editorial Dunken. Corrientes. Argentina.

Domínguez S., C. 2010. "Las políticas públicas en materia de empleo juvenil. Hacia un enfoque integral, focalizado y que reconozca la heterogeneidad de las trayectorias juveniles". En García Vior, A. Trabajo de Jóvenes y Menores. El acceso al primer empleo y la prohibición del trabajo infantil. Corrientes. Argentina.

Dubra, D. 2010. "El trabajo familiar de los menores. Los menores y adolescentes como auxiliares del trabajador (art. 28 L.C.T.)". En García Vior, A. Trabajo de Jóvenes y Menores. El acceso al primer empleo y la prohibición del trabajo infantil. Corrientes. Argentina.

Litterio, L. 2010. El trabajo infantil y adolescente en la Argentina. Las normas y la realidad. Editorial Errepar. Buenos Aires. Argentina.

Litterio, L. 2013. "Nuevos instrumentos para la fiscalización del trabajo infantil y adolescente en empresas". Revista Derecho del Trabajo $N^{\circ} 4$ (abril de 2013). Editorial La Ley. Corrientes. Argentina.

Litterio, L. 2010. "Sobre la prohibición del trabajo infantil y la elevación de la edad mínima para comenzar a trabajar: desafíos para la Argentina". En García Vior, A. Trabajo de Jóvenes y Menores. El acceso al primer empleo y la prohibición del trabajo infantil. Corrientes. Argentina.

Livellara C. 2010. "El contrato de trabajo de aprendizaje luego de las reformas de la Ley 26390". En García Vior, A. Trabajo de Jóvenes y Menores. El acceso al primer empleo y la prohibición del trabajo infantil. Corrientes. Argentina.

Rial, N. 2009. Prohibición del Trabajo infantil y Protección del Trabajo Adolescente. Ministerio de Trabajo, Empleo y Seguridad Social. Unicef. Buenos Aires. Argentina.

Silva, M. 2010. "Federalismo, políticas públicas y trabajo infantil". En Zarate H.; Ramírez Brachi D. VIJornadas de Comunicaciones Cientificas de la Facultad de Derecho y Ciencias Sociales y Políticas-UNNE. Editorial Moglia. Corrientes. Argentina.

Silva, M.; Boleso, H. Trabajo infantil y educación. 2010. Editorial Moglia. Corrientes. Argentina. 
Silva, M. Trabajo infantil y salud: aportes a la construcción del conocimiento. Editorial Académica Española. Saarbrucken. Germany.

Silva, María Alejandra. "Desigualdades y trabajo infantil"; Revista Prospectiva, $N^{\circ} 15$, Facultad de Trabajo Social, Universidad del Valle de Cali, Colombia.

Silva, M. "Niñez en situación de vulnerabilidad: ¿olvidada por las políticas?". Revista $\mathrm{N}^{\circ} 10$ "Instituciones y Procesos Gubernamentales V", Revista de Ciencia Política: de la Ciudad de Buenos Aires a la Aldea Global, Buenos Aires, Argentina.

Silva, María Alejandra, Trabajo infantil rural en Argentina [Rural work by minors en Argentina]. Noviembre 30-1-11.

Silva, María Alejandra, "Federalismo, desigualdades y niñez". Publicada en "Boletín Enredando" (Reproduce el paper presentado en V Jornadas Internacionales sobre Estado y Sociedad/UBA). Publicado por Nodo Tau, Rosario, 21-06-10.

Silva, M. "Agricultura familiar y desarrollo sustentable: una mirada desde las políticas protectoras del trabajo decente y saludable". Publicada en "Intercambios", Año 10, Número 116. Se reproduce el paper presentado en IV Congreso Internacional de la Red SIAL, organizado por INTA Balcarce/2008, Centro Latinoamericano para el desarrollo rural (RIMISP), Publicado en Chile, noviembre 2010.

Silva, M. "El trabajo infantil: ¿es posible abordarlo desde la historia de vida?; Revista Educación y Pedagogía, Facultad de Educación, Medellín (Colombia). En prensa.

Silva, M. "El trabajo infantil rural en la agenda pública del desarrollo sustentable". Revista Espacio Abierto, Maracaibo (Venezuela). En prensa.

Silva, M. "El abordaje del trabajo infantil desde la indagación de la familia: un aporte a las políticas públicas". Revista Ciencia Política, Departamento de Ciencia Política de la Universidad Nacional de Colombia (en referato).

Silva, M. 2012, "Trabajo infantil y salud: balances y desafíos". En: Mariela Macri y Claudia Uhart (comp.), Trabajos infantiles e infancias: investigaciones en territorio (2005-2010), Editoriales La Crujía, Buenos Aires.

Surt, S.; De Bianchetti A. 2012, "Niñez Vulnerable. Estrategias de Intervención". 
En Zárate H.; Ramírez Brachi D. VIII Jornadas de Comunicaciones Cientificas de la Facultad de Derecho y Ciencias Sociales y Políticas-UNNE. Editorial Moglia. Corrientes. Argentina.

Virgili, N.; Virgili C. 2010, "Los adolescentes y el trabajo". En García Vior, A. Trabajo de Jóvenes y Menores. El acceso al primer empleo y la prohibición del trabajo infantil. Corrientes. Argentina. 